\title{
Eine Herausforderung für die Forschung
}

\section{Wachstumsunabhängigkeit}

\author{
Für die Transformation hin zu einer wachstums- \\ unabhängigen Gesellschaft kann Wissenschaft \\ gezielt Freiräume für politisches Handeln und \\ gesellschaftliche Entwicklung aufzeigen. Dafür \\ sind substanzielle Impulse durch die Forschungs- \\ förderung notwendig. \\ Von David Hofmann, Daniel Eichhorn, \\ Thomas Korbun und Kora Kristof
}

E ine zentrale Aufgabe von Wissenschaft mit transformativem Anspruch besteht darin, neue Möglichkeitsräume für gesellschaftliches Handeln zu eröffnen. Die Abhängigkeit moderner Industriegesellschaften vom Wirtschaftswachstum und die starke politische Orientierung darauf haben das Potenzial, die Einhaltung globaler Nachhaltigkeitsziele zu gefährden, wenn es nicht gelingt, rechtzeitig eine hinreichend starke absolute Entkopplung von Wachstum und Umweltbelastung zu erreichen. Daher besteht aus dem Vorsorgemotiv heraus die Notwendigkeit, Alternativen zu auf Wachstum basierenden Strukturen und Entwicklungen sowie mögliche Transformationspfade in Richtung einer wachstumsunabhängige(re)n Gesellschaft zu entwickeln und zur Diskussion zu stellen.

Mit welchen Instrumenten könnte die Realisierung einer wachstumsunabhängigen Wirtschaft und Gesellschaft gelingen? Welche ökologischen, ökonomischen und sozialen Konsequenzen hätte dies? Diese zentralen Fragen können derzeit aus wissenschaftlicher Sicht nicht abschließend beantwortet werden [1]. Die Debatten um Wachstumsunabhängigkeit und Postwachstum wurden bisher insbesondere in Teilen der Zivilgesellschaft und erst in Ansätzen in wissenschaftlichen Arenen geführt. Somit besteht ein großer Bedarf an grundlegenden und explorativen inter- und transdisziplinären wissenschaftlichen Analysen, empirischen Arbeiten und praktischen Experimenten zu den derzeit wachstumsabhängig aufgesetzten Strukturen und Rahmenbedingungen und möglichen wachstumsunabhängigen Alternativen.

Zu diskutieren ist auch, ob das gegenwärtige Wissenschaftssystem in hinreichendem Maße über die nötigen Voraussetzungen und inter- und transdisziplinären Kompetenzen für die notwendigen gesellschaftlichen Suchprozesse verfügt (siehe die Debatte um transformative Ökonomik im Schwerpunkt von ÖkologischesWirtschaften 32/2). Aus unserer Sicht sollte auch die Wissenschaft eigene Pfadabhängigkeiten und wirk- mächtige Narrative kritisch reflektieren. Hierzu zählen eine starke Fokussierung auf technische Ansätze zur Lösung von komplexen gesellschaftlichen Nachhaltigkeitsproblemen, die fehlende Ausschöpfung interdisziplinärer Potenziale oder eine Ausrichtung der wirtschaftswissenschaftlichen Politikberatung auf Konzepte, die voraussetzen, dass weiteres wirtschaftliches Wachstum wünschenswert und möglich ist.

Zukünftige Forschungsvorhaben sollten klären, ob es möglich und wünschenswert ist, gesellschaftlich relevante Bereiche wie Sozialversicherungssysteme, Steuersystem, Beschäftigungspolitik oder Infrastrukturen wachstumsunabhängig(er) zu gestalten. Zwar konnten bereits verschiedene Potenziale für wachstumsunabhängige Lösungen identifiziert werden (Petschow et al. 2018), doch bezüglich der umfassenden wissenschaftlichen Bewertung der Nachhaltigkeitswirkungen, der konkreten Ausgestaltung und systemischen Wirkungen von wachstumsunabhängigen Strategien besteht erheblicher Forschungsbedarf. Dabei sollte umfassend untersucht werden, ob und unter welchen Bedingungen es sinnvoll sein kann, konkrete Wachstumstreiber gezielt abzuschwächen. Aufgrund noch weniger untersuchbarer Fälle gibt es kaum empirische Arbeiten zu vorgeschlagenen Politikinstrumenten wie hohen und umfassenden Ökosteuern oder substanziellen Arbeitszeitverkürzungen. International vergleichende empirische Studien und experimentelle Analysen könnten die empirische Basis verbessern.

\section{Wie verändert sich der Wohlstandsbegriff?}

Eine weitere zentrale Fragestellung ist, wie und unter welchen Bedingungen sich Werte und Kultur wandeln. Wie verändern sich der Wohlstandsbegriff und die gesellschaftliche Bedeutung von Wachstum? Wie finden suffiziente Lebensstile stärkere Verbreitung? Trotz dynamischer Forschungsaktivitäten in den vergangenen Jahren (unter anderem in der internationalen Transitionsforschung), besteht zu diesen Fragen noch erheblicher Forschungsbedarf.

Um gesellschaftlich und wissenschaftlich relevante Antworten auf diese komplexen Fragen entwickeln zu können, muss der Austausch mit anderen gesellschaftlichen Debatten und zwischen Disziplinen stärker als bisher gelingen. So können die Debatten um Wachstumsunabhängigkeit und Postwachstum in beidseitige fruchtbare Beziehungen gesetzt werden $\mathrm{zu}$ aktuellen, in der breiten Öffentlichkeit geführten Debatten zur Zukunft der Arbeitswelt, des Sozialstaates, zu Verteilungsfragen, zur Digitalisierung oder zur Förderung gesellschaftlicher und ökonomischer Teilhabe in Regionen im Struktur- 
wandel. Interessante Bezüge ergeben sich zudem zu wissenschaftlichen Fachdiskursen wie zur Resilienz sozio-technischer Systeme oder zu Wohlfahrtsindikatoren als Alternative zum Wachstum des BIP als dominante gesellschaftliche Zielgröße. Produktiv könnte auch die Verbindung von makroökonomischen Forschungen und Nachhaltigkeitsforschung sein.

Um neue Pfade der gesellschaftlichen Entwicklung zu explorieren, die auch die empirische Basis für wissenschaftliche Analysen verbreitern, sollten zudem gezielt Freiräume für die Entwicklung und Erprobung nachhaltiger Lebens- und Wirtschaftsweisen geschaffen und bestehende Hürden abgebaut werden. Hierfür bieten sich transdisziplinäre Projekte besonders an. So könnten etwa in regionalen Reallaboren mögliche Lösungen für wachstumsunabhängige Strukturen und den Übergang dorthin untersucht werden. Das kann auch Antworten bieten auf die Herausforderungen, die wirtschaftliche und gesellschaftliche Strukturwandelsprozesse wie beispielsweise der Kohleausstieg aufwerfen. Potenziale für eine wachstumsunabhängigere und sozial inklusive Regionalentwicklung könnten in alternativen Formen des Wirtschaftens oder in sozialen Innovationen wie commons-basierten Produktions- und Konsumweisen liegen. Ebenso könnte untersucht werden, wie unterschiedliche Auffassungen zum guten Leben wirken, wie sich Bedürfnisse wandeln und ob neue Wohlfahrtsindikatoren die nötige Akzeptanz und Handlungsrelevanz entfalten können. Auch könnten die Wechselbeziehungen von regionalen Ansätzen des Wirtschaftens mit nationalstaatlichen und internationalen Ebenen analysiert werden.

Bei der Suche nach Antworten zu den hier skizzierten Fragestellungen sind auch grundsätzliche methodische Herausforderungen zu meistern. So besitzen zum Beispiel ex-post-Evaluierungen von in der Vergangenheit eingesetzten Instrumenten nur eine begrenzte Aussagekraft hinsichtlich der zu erwartenden Effekte bei einer künftigen Implementierung mit deutlich größerer Eingriffstiefe. Die bisher bekannten Ansätze zur Verwirklichung einer stärkeren Wachstumsunabhängigkeit sind bezüglich ihrer Wirksamkeit als marginal einzuschätzen (Petschow et al. 2018). Grundlegende Reformansätze werden bisher nur im Rahmen kleinerer Experimente verfolgt. Für besser belastbare Aussagen über ihre Generalisierbarkeit und potenzielle Wirksamkeit sind damit neuartige methodische Ansätze zu entwickeln.

\section{Impulse durch Forschungspolitik notwendig}

Auch durch die Enquete-Kommission „Wachstum, Wohlstand, Lebensqualität" des Deutschen Bundestags wurde bereits grundsätzlicher Forschungsbedarf identifiziert (siehe Haake et al. 2013). Mögliche Forschungsfragen für die sozial-ökologische Forschung zu wachstumsunabhängigen Pfaden liegen vor (BM B F 2018). Angesichts der grundsätzlichen gesellschaftlichen Bedeutung und des disziplinenübergreifenden Charakters der Thematik braucht es erstens substanzielle Impulse für die Entwicklung und Erprobung wachstumsunabhängiger Lösun- gen durch die Forschungsförderung, jenseits der disziplinären Logiken des Wissenschaftssystems. Transdisziplinäre Projekte könnten dabei die Nutzbarkeit der Ergebnisse für die wissenschaftliche Politikberatung verbessern. Zweitens sollte ein wissenschaftlich begleitetes „Erprobungsprogramm“ ermöglichen, neue Umsetzungsvorschläge beispielsweise in Reallaboren zu "testen“. Dabei sollten transformative Elemente in der Innovationspolitik gestärkt werden, die dazu beitragen, soziale Experimente und gesellschaftliche Lernprozesse in verschiedenen Handlungsfeldern zu fördern, sodass bisher unbekannte Pfade zu einer nachhaltigen Entwicklung ermöglicht werden. Parallel gilt es drittens, die empirische Basis für weitere Forschungsvorhaben zu verbreitern und die Entwicklung von Methoden zur Wirkungsabschätzung sowie zur Skalierung und Generalisierung von Experimenten voranzutreiben.

\section{Anmerkung}

[1] Im Folgenden beziehen wir uns auf Ergebnisse des Projektes „Ansätze zur Ressourcenschonung im Kontext von Postwachstumskonzepten“, das von IÖW, RWI und Wuppertal Institut im Auftrag des Umweltbundesamtes durchgeführt wurde. Ebenso auf aktuelle forschungspolitische Empfehlungen wie sie im Agendaprozess Sozial-ökologische Forschung des Bundesministeriums für Bildung und Forschung entwickelt wurden.

\section{Literatur}

Bundesministerium für Bildung und Forschung (BMBF) (Hrsg.) (2018): Themenpapier Sozial-ökologische Pfade zu einer wachstumsunabhängigen Gesellschaft.

Haake, H. et al. (2013): Zum Bericht der Enquete-Kommission Wachstum, Wohlstand, Lebensqualität - Viele Fragen und wenig Lösungen. In: ÖkologischesWirtschaften 28/3: 23-24.

Petschow, U./aus dem Moore, N./Pissarskoi, E./Korfhage, T./Lange, S./ Schoofs, A./Hofmann, D. (2018): Gesellschaftliches Wohlergehen innerhalb planetarer Grenzen: Der Ansatz einer vorsorgeorientierten Postwachstumsposition. UBA Texte 89/2018.

\section{AUTOR/INNEN + KONTAKT}

David Hofmann ist Referent am Institut für ökologische Wirtschaftsforschung (IÖW). Thomas Korbun ist Wissenschaftlicher Geschäftsführer am IÖW. Institut für ökologische Wirtschaftsforschung, Potsdamer Str. 105, 10785 Berlin.

Tel.: +49 30 884594-0, E-Mail: david.hofmann@ioew.de, thomas.korbun@ioew.de, Website:www.ioew.de

Daniel Eichhorn ist wissenschaftlicher Mitarbeiter am Umweltbundesamt im Fachgebiet Grundsatzfragen, Nachhaltigkeitsstrategien und -szenarien, Ressourcenschonung. Er war Fachbetreuer des Projekts „Ansätze zur Ressourcenschonung im Kontext von Postwachstumskonzepten“.

Dr. Kora Kristof ist Leiterin der Abteilung „Nachhaltigkeitsstrategien, Ressourcenschonung und Instrumente" des Umweltbundesamtes.

Umweltbundesamt, Wörlitzer Platz 1 06844 Dessau-Roßlau. Tel.: +49 340 21032652, E-Mail: daniel.eichhorn@uba.de, kora.kristof@uba.de, Website: www.uba.de
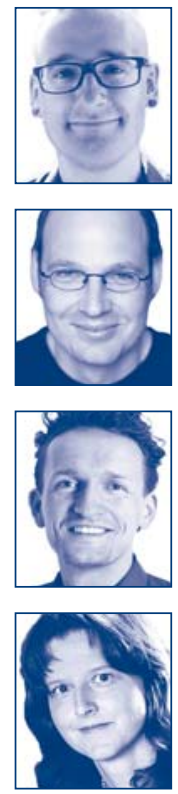